\title{
Preparation, characterization and evaluation of some metallic lube oil additives
}

\author{
Nehal S. Ahmed ${ }^{1)}$ Amal M. Nassar ${ }^{1)}$, Yasser K. Abd el menem ${ }^{2)}$ and \\ Reham I. El- shazly ${ }^{1)^{*}}$ \\ 1Department of Petroleum Applications, Egyptian Petroleum Research Institute, Nasr City, Cairo, Egypt. \\ 2Permanent address: Chemistry Department, Faculty of Science, Menofia University, Shebin el koum, Menofia, \\ Egypt. \\ 2Current address: Taif university, Saudia Arabia
}

\begin{abstract}
All mechanical equipment suffers from wear due to the time of use and exposure to contaminants that can damage its movable parts. One way to extend the life time of this equipment is through the use of suitable lubricating oil with different additives. Detergents, especially basic detergents, contain reserve base that will neutralize the acids to form salts. While this decreases the corrosive tendency of the acids formed at high temperatures. Dispersants also disperse sludge formed in engine operated at low temperature. In the present work, different metallic detergent/dispersant additives will be prepared via reaction of dodecyl phenol with calcium hydroxide in different ratios and different amounts of sulfur. The structures of the prepared compounds were confirmed by Fourier transform infrared spectroscopy (FTIR), Proton Nuclear Magnetic Resonance $\left(H^{I}\right.$ NMR) and molecular weight is determined. All the prepared compounds were found to be soluble in lubricating oil. The efficiency of the prepared compounds as antioxidant and detergent/dispersant additives for lubricating oil was investigated. It was found that the additives have excellent power of dispersion and detergency and the efficiency as antioxidant increases by increasing the sulfur content up to $1.5 \%$ and increases by increasing the percentage of metal in the additives.
\end{abstract}

Key words: Lubricating Oil Additives, Basic and Over basic Detergents/Dispersants, Calcium Phenate, Sulfonate and Salicylate, Acid neutralization, Reverse micelle.

\section{Introduction}

Base stock oil properties are insufficient to meet the current demands of engine efficiency (low friction) durability, longer drain times and emissions regulations. Therefore, the lubricating oil must meet specific requirements. It must have an optimal balance of light and heavy oil components to lubricate at high temperature [1] it should not produce deposits (carbon soot and other) on moving parts. In addition, the oil should provide good protection of moving parts at high speed under deceleration of the engine [2]. Additives are therefore blended with base oils to perform two base functions. To minimize destructive processes and to confirm beneficial properties .Almost all lubricants contain additives to enhance their performance in amounts ranging between $5-25 \% \mathrm{wt}[3,4]$.

Additives are synthetic chemicals used to improve different lube parameters: they can boost existing properties, eliminate adverse characteristics, or introduce new properties in the base oil [1]. Main functions of additives are: antioxidants, viscosity modifiers, pour point depressants, detergents, dispersants, antifoam agents, antiwears, friction modifiers, and antirust [5]. Various additives have been developed to control the acidity of the products of sulphurous combustion of dirty fuel and to prevent agglomeration of soot from combustion and wear particles. The agglomeration of particles can be very destructive to engines since it blocks the oil supply pipelines or even the filters. Additives which prevent the development of all these detrimental effects are known in the literature as either 'detergents' or 'dispersants'. The latter term, however, is more accurate. The primary functions of these additives are:

. to neutralize any acids formed during the burning of fuel,

. to prevent lacquer and varnish formation on the operating parts of the engine,

. to prevent the agglomeration of particles and carbon deposits which may impede the oil ways.

There are two types of detergent/dispersant additives (d/d additives): mild d/d additives and over-based or alkaline $\mathrm{d} / \mathrm{d}$ additives. Mild d/d additives are often composed of simple hydrocarbons or ashless compounds (i.e., when the compound is burnt no oxides are left, since organic compounds burn to $\mathrm{CO}_{2}$ and water). The function of these additives is to disperse soot (carbon) and wear particles. Over-based d/d additives are calcium, barium or zinc salts of sulphonic, phenol or salicylic acids. Over-based means that an excess of alkali is used in the preparation of these additives. 
Detergents and dispersants constitute $2-15 \%$ of the additives [6]. These compounds keep oil-insoluble combustion byproducts in suspension and prevent the agglomeration of the oxidation products into solid particles. Commonly, these chemicals are metal-containing compounds with a large oleophilic hydrocarbon portion and a polar hydrophilic region. Polar group can link lube contaminants, while the tail section act as a solubilizer in the base oil.

The surfactants used to prepare overbased detergents have an amphiphilic structure. The polar headgroup, which typically binds to a calcium, magnesium or sodium metal cation, may be a benzene sulfonate, phenate, salicylate or phosphonate [7]. The surfactants act as a dispersed agent, while the metal carbonate acts as a neutralizing agent, and the diluent oil acts as a compatible agent. The term [8-10] "over based" demonstrates that the quantity of colloidal metal carbonate in the particle cores is greater than that is needed to neutralize the surfactant acid, otherwise it is described as "neutral" or "low basicity", indicating that the over based detergents have a greater acid neutralizing capacity than their neutral salts. In the present work, different metallic detergent/dispersant additives will be prepared via reaction of dodecyl phenol with calcium hydroxide in different ratios. The structures of the prepared compounds were proved using Fourier transform infrared spectroscopy (FTIR), Proton Nuclear Magnetic Resonance ( $\left.\mathrm{H}^{1}-\mathrm{NMR}\right)$ and molecular weight is determined. The efficiency of the prepared compounds as antioxidant \& detergent/dispersant additives for lubricating oil was investigated.

\section{Experimental}

\subsection{Sulfurization and neutralization of dodecyl phenol:}

0.1 mole of dodecyl phenol and 0.05 mole of calcium hydroxide with variable amounts of sulfur $(0$, $0.025,0.05,0.1,0.15$, and 0.2$)$ mole were mixed in three necks round bottom flask equipped with a mechanical stirrer, reflux condenser and thermometer. The reaction mixture was maintained at temperature $130^{\circ} \mathrm{C}$. Then the temperature was raised gradually to $160^{\circ} \mathrm{C}$ for 2 hours. And it was raised gradually to $190^{\circ} \mathrm{C}$ for 2 hours. (33\% of the reactant volume) of white oil are added. Then we obtained the products (A, B, C, D, E and F).

\subsection{Basing and overbasing process:}

Sulfurized calcium salt of dodecyl phenol (E) was mixed with $30 \mathrm{gm}$. of toluene and $9.8 \mathrm{gm}$. of methanol then the excess of calcium hydroxide was added by a percent of 1mole of calcium phenate to 1 mole of calcium hydroxide (basic salt $\left(\mathrm{E}_{2}\right)$ ) and 1 mole of calcium phenate to 2.5 mole of calcium hydroxide (over basic salt $\left(\mathrm{E}_{3}\right)$ ). The mixture was stirred in a three neck flask, $\mathrm{CO}_{2}$ was inserted with a rate of $50 \mathrm{ml} / \mathrm{min}$. for half an hour. Then $10 \mathrm{gm}$. of white oil was added as a diluent. Solvent was removed using rotary evaporator at $90^{\circ} \mathrm{C}$. Therefore we have two different products, the designation of the prepared compounds are shown in Table (1).

Table (1): The designation of prepared compounds.

\begin{tabular}{|l|l|}
\hline Abbreviation & Prepared Compounds \\
\hline A & Neutral non sulfurized calcium dodecyl phenate $(\% \mathrm{~S}=0)$ \\
\hline B & Neutral sulfurized calcium dodecyl phenate $(\% \mathrm{~S}=0.25)$ \\
\hline $\mathrm{C}$ & Neutral sulfurized calcium dodecyl phenate $(\% \mathrm{~S}=0.5)$ \\
\hline $\mathrm{D}$ & Neutral sulfurized calcium dodecyl phenate $(\% \mathrm{~S}=1)$ \\
\hline $\mathrm{E}$ & Neutral sulfurized calcium dodecyl phenate $(\% \mathrm{~S}=1.5)$ \\
\hline $\mathrm{F}$ & Neutral sulfurized calcium dodecyl phenate $(\% \mathrm{~S}=2)$ \\
\hline $\mathrm{E}_{2}$ & Basic sulfurized calcium dodecyl phenate $(\% \mathrm{~S}=1.5)$ \\
\hline $\mathrm{E}_{3}$ & Over basic sulfurized calcium dodecyl phenate $(\% \mathrm{~S}=1.5)$ \\
\hline
\end{tabular}

\subsection{Characterization of the prepared compounds:}

\subsubsection{Infrared Spectroscopic Analysis:}

The prepared compounds were characterized by using F.T.I.R. Spectrometer Model Type MattsonInfinity Series Bench top, which made in USA.

\subsubsection{Determination of Molecular Weights:}

The molecular weights of different prepared compounds were determined by physical method known as

"cryscopic method", it is based on the principle that the freezing point of pure solvent such as benzene changes

on contamination with any foreign material for example: crude oil sample.

The average molecular weight is measured according to the following equation:

$$
\text { M.wt }=\frac{\text { const. } K}{\Delta T} * \frac{\text { wt. of crude oil }(\mathrm{g} .)}{\text { wt. of solvent }(\mathrm{g.})} * 1000
$$

Where: $\Delta \mathrm{T}$ is the decrease in the freezing point.

$\mathrm{K}$ is a constant depending on the type of solvent. 
e.g $\mathrm{K}$ for benzene $=5.12$

\subsubsection{Proton Magnetic Resonance Analysis:}

The prepared compounds were characterized by ${ }^{1}$ H-N.M.R. spectroscopy. Using ${ }^{1}$ H-N.M.R. type [300 M.Hs. spectrophotometer W-P-300, Bruker].

\subsection{Evaluation of the Prepared Compounds as Lube Oil additives:}

\subsubsection{As Antioxidants.}

The lube oil samples as well as its blends with $2 \%$ by weight of each of the prepared additives were subjected to sever oxidation condition in the presence of copper and iron strips at $165.5^{\circ} \mathrm{C}$ for $72 \mathrm{hrs}$ using the Indiana test method of oxidation [11]. The oxidation stability of the lube oil blends were determined by taking samples at $24 \mathrm{hrs}$, intervals to $72 \mathrm{hrs}$. These samples were tested for:

1- Variation of viscosity ratio $\mathrm{V} / \mathrm{V}_{\mathrm{o}}$.

The variation of viscosity ratio (V/Vo) has been determined using IP 48/86 method, where:

$\mathrm{V}=$ Kinematic viscosity at $40^{\circ} \mathrm{C}$ of sample after oxidation.

Vo $=$ Kinematic viscosity at $40{ }^{\circ} \mathrm{C}$ of sample before oxidation.

2- $\quad$ Change in total acid number ( $\Delta$ T.A.N.).

The change has been calculated according to IP $177 / 83$ method, where.

$\Delta$ T.A.N. = (total acid number of sample after oxidation - total acid number of sample before oxidation).

3- $\quad$ Optical density using infrared techniques.

The infrared spectra of oxidized oils have been determined in the range of the carbonyl group absorbance (1500$1900 \mathrm{Cm}^{-1}$ ). The spectra have been superimposed upon that of unoxidized oil. The absorbance (A) has been calculated according to

$\mathrm{A}=\log \mathrm{I} / \mathrm{I}_{\mathrm{o}}$

Where: I is \% transmittance of the oil after oxidation and $\mathrm{I}_{0}$ is the transmittance of the oil before oxidation.

\subsubsection{As Detergents / Dispersants.}

\section{Spot method}

Drops were taken from the samples being oxidized in the Indiana test after $24 \mathrm{hrs}$. intervals of oxidation and up to $72 \mathrm{hrs}$. to make spots on special filter paper (Durieux 122) and the dispersancy of the samples were measure as follows $[11,12]$ :

$$
\% \text { Dispersancy }=\frac{\text { Diameter of the black spot }}{\text { Diameter of the total spot }} \times 100
$$

The efficiency of dispersants has been classified as follows:

- Up to $30 \%$ : no dispersancy.

- 30-50\%: medium dispersancy.

- 50-60\%: good dispersancy.

- 60-70\%: very good dispersancy.

- Above $70 \%$ : excellent dispersancy.

\section{Determination of potential detergent / dispersant efficiency (PDDE):}

Two factors are necessary to determine PDDE.

a) Detergent index :-

This method based on centrifugation. A suspension was prepared from the base oil containing $1.5 \%$ additive and $2 \%$ carbon black. This suspension was shared by ultrasonic equipment. Afterwards, the blend was diluted with kerosene and the mixture was centrifuged for $30 \mathrm{~min}$. The blend was examined in photometrical equipment at $530 \mathrm{~nm}$. Detergent index (DI) determined on the basis of the intensity of tran smitted light:

$$
\mathrm{DI}=\left(\mathrm{I}_{1} / \mathrm{I}_{0}\right) \times 100
$$

Where: $I_{1}$ is the intensity of the light after transmission through the blend containing carbon black; $I_{0}$ is the intensity of the light transmitted through the blend free of carbon black.

\section{b) Washing efficiency :-}

The washing efficiency (WE) was measured by Zaslavskii's modified method based on thin layer chromatography. The efficiency was evaluated within $0-125 \mathrm{~mm}$. The results of numerous potential experiments attested that these two methods were suitable to estimate the potential of detergent/dispersant efficiency (PDDE) in oil solutions:

$$
\mathrm{PDDE}=\frac{\mathrm{DI}+\mathrm{WE}}{225} \times 100
$$

org $\quad 58 \mid$ Page


Where DI is the detergent index (\%), WE is the washing efficiency $(\mathrm{mm}), 225$ is the maximum value of DI + WE $($ DI $\max =100$, WE $\max =125)[13]$.

\subsection{Sulfurization \& neutralization of dodecyl phenol:}

\section{Results and Discussion}

Dodecyl phenol reacts with calcium hydroxide and different ratio of sulfur to give neutral sulfurized calcium salt of dodecyl phenol (A, B, C, D, E and F). Sulfurization and neutralization of dodecyl phenol illustrated in scheme (1) as follows:

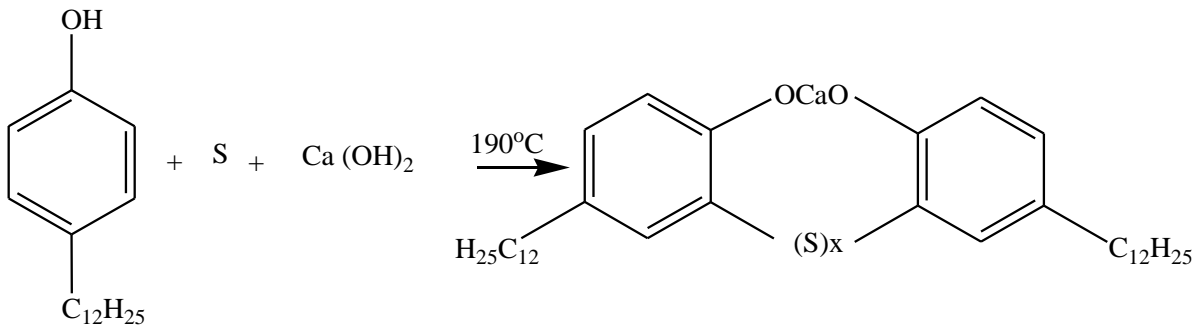

Where $\mathrm{X}$ is a variable $(0,0.025,0.05,0.1,0.15$, and 0.2$)$ mole Scheme (1): Reaction of formation of neutral sulfurized calcium phenate.

Table (2): The physico-chemical characteristics of the prepared compounds.

\begin{tabular}{|l|l|l|l|l|l|l|}
\hline Characteristics & A & B & C & D & E & F \\
\hline Mean M.wt & 420 & 433.68 & 494.62 & 911 & 638.89 & 710 \\
\hline Theoretical M.wt & 562 & 592 & 632 & 672 & 712 & 744 \\
\hline T.B.N & 35.5 & 37.7 & 40.75 & 47 & 55 & 57.6 \\
\hline Calcium wt\% & 2.98 & 3.12 & 3.14 & 5.22 & 4.05 & 2.96 \\
\hline Sulfur wt\% & 0 & 1.685 & 2.728 & 4.122 & 5.586 & 6.203 \\
\hline Carbon wt\% & 77.069 & 76.8 & 77.44 & 73.96 & 75.23 & 74.34 \\
\hline Hydrogen wt\% & 8 & 8.02 & 7.9 & 7.98 & 6.73 & 7 \\
\hline Oxygen wt\% & 14.931 & 11.095 & 8.792 & 8.718 & 8.404 & 9.497 \\
\hline
\end{tabular}

From the results of molecular weight, it is clear that the experimental results differ from the theoretical results, which may be due to formation of compounds as bi-products other than the expected compounds. The general formulas of these compounds are:<smiles>Cc1ccc(C(=O)O)cc1</smiles>

$\mathrm{C}_{12} \mathrm{H}_{25}$

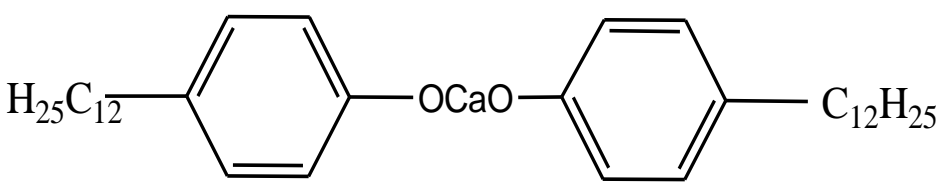

And the general formulas of the prepared additives are shown in Table (3)

Table (3): The general formula of the additives prepared

\begin{tabular}{|l|l|}
\hline Sample & experimental formula \\
\hline A & $\mathrm{C}_{26.96} \mathrm{H}_{33.6} \mathrm{O}_{3.9} \mathrm{Ca}_{0.31}$ \\
\hline B & $\mathrm{C}_{27.76} \mathrm{H}_{34.78} \mathrm{~S}_{0.23} \mathrm{O}_{10} \mathrm{Ca}_{0.34}$ \\
\hline C & $\mathrm{C}_{31.9} \mathrm{H}_{39.07} \mathrm{~S}_{0.42} \mathrm{O}_{2.7} \mathrm{Ca}_{0.39}$ \\
\hline D & $\mathrm{C}_{58.79} \mathrm{H}_{72.69} \mathrm{~S}_{1.17} \mathrm{O}_{4.96} \mathrm{Ca}_{1.19}$ \\
\hline
\end{tabular}




\begin{tabular}{|l|l|}
\hline E & $\mathrm{C}_{40} \mathrm{H}_{43} \mathrm{~S}_{1.113} \mathrm{O}_{3.74} \mathrm{Ca}_{0.7}$ \\
\hline F & $\mathrm{C}_{43.98} \mathrm{H}_{49.7} \mathrm{~S}_{1.37} \mathrm{O}_{4.189} \mathrm{Ca}_{0}$ \\
\hline
\end{tabular}

The infrared spectrum of product (C) is shown in Fig (1), which illustrate the following:

1) Disappearance of $\mathrm{OH}$ group band (at $\left.3300 \mathrm{Cm}^{-1}\right)$ in the prepared compound $(\mathrm{C})$.

2) Appearance of C-S peak at $700 \mathrm{Cm}^{-1}$ in the prepared compound (C).

3) Appearance of S-S peak at $550 \mathrm{Cm}^{-1}$ in the prepared compound (C).

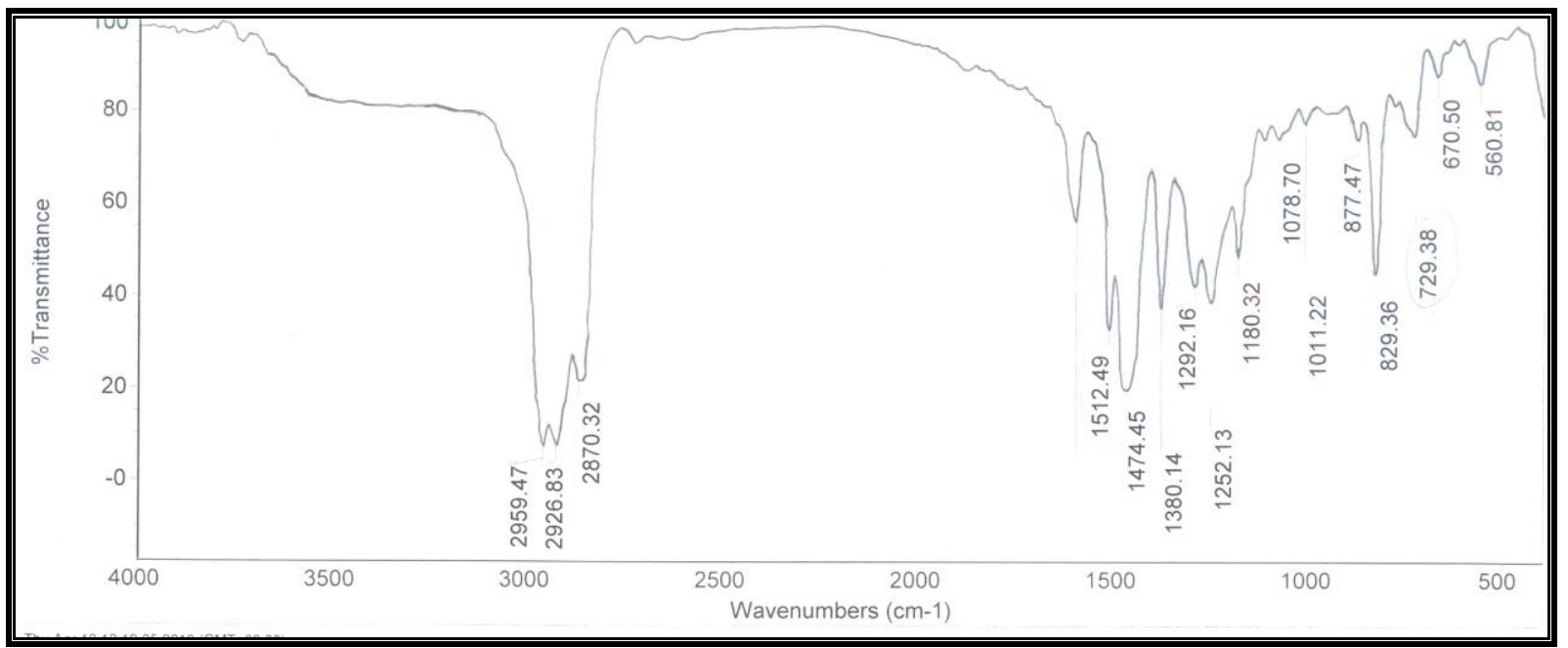

Figure (1): Infrared spectrum of compound (C)

The $\mathrm{H}^{1} \mathrm{NMR}$ spectra of compounds (E, $\mathrm{E}_{2}$ and $\mathrm{E}_{3}$ ) are shown in Figs. (2-4), which illustrate the following:

1. A peak appears at $8-9 \mathrm{~nm}$ in case of compound (E), which indicates the presence of phenolic OH. This peak disappears in case of compound $\left(\mathrm{E}_{2}\right)$ and $\left(\mathrm{E}_{3}\right)$, this may be due to the presence of percentage of unreacted dodecyl phenol in the preparation of the neutral compound.

2. A peak appears at $0-2 \mathrm{~nm}$ in case of three compounds $\left(\mathrm{E}, \mathrm{E}_{2}\right.$ and $\mathrm{E}_{3}$ ) which indicates the presence of alkyl hydrogen.

3. A peak appears at $6-8 \mathrm{~nm}$ in case of three compounds $\left(E, E_{2}\right.$ and $\left.E_{3}\right)$ which indicates the presence of benzene ring.

4. A peak appears at 3-4 $\mathrm{nm}$ in case of three compounds $\left(\mathrm{E}, \mathrm{E}_{2}\right.$ and $\left.\mathrm{E}_{3}\right)$ which indicates the presence of $\mathrm{Ar}-\mathrm{CH}$ group. 


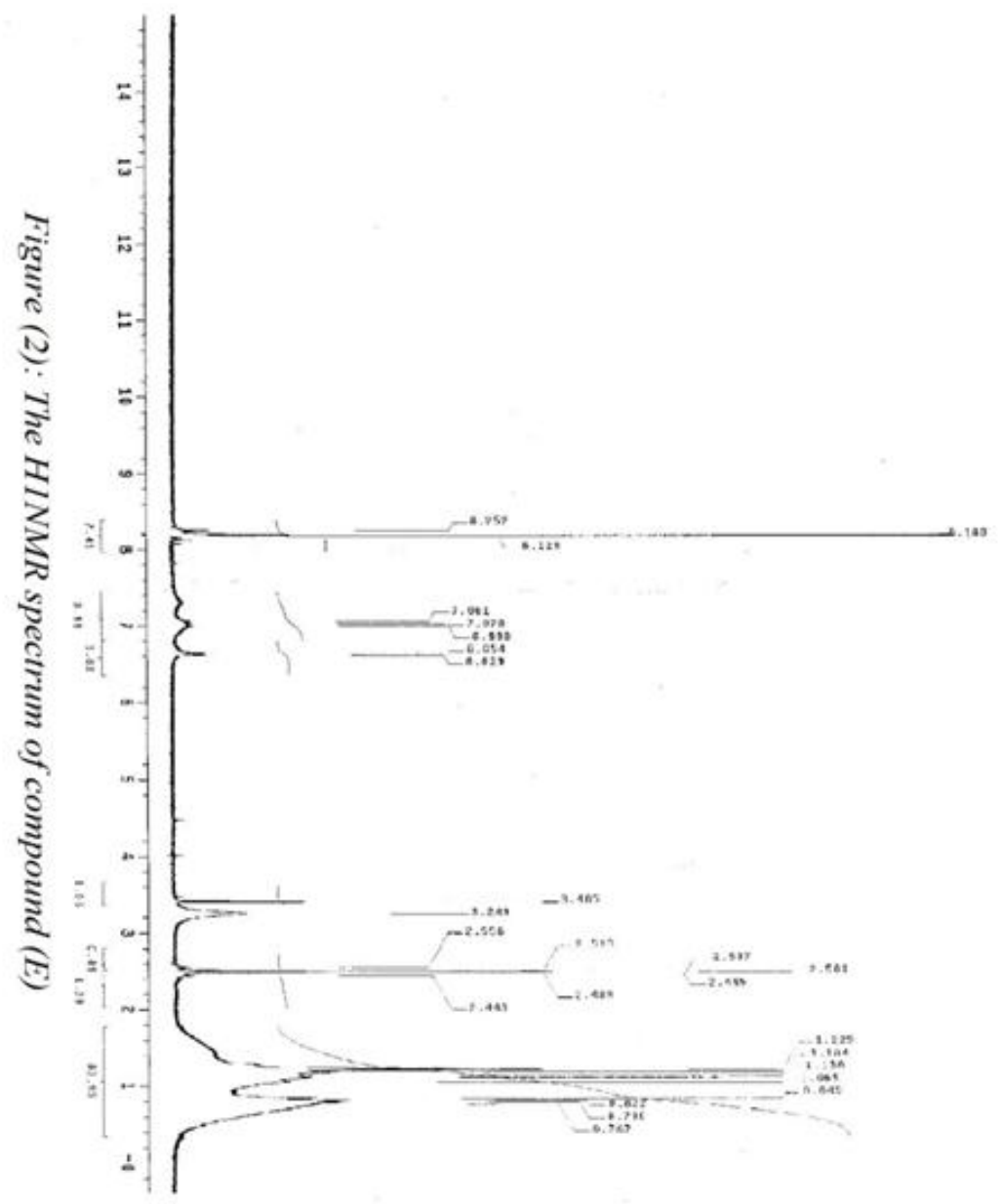




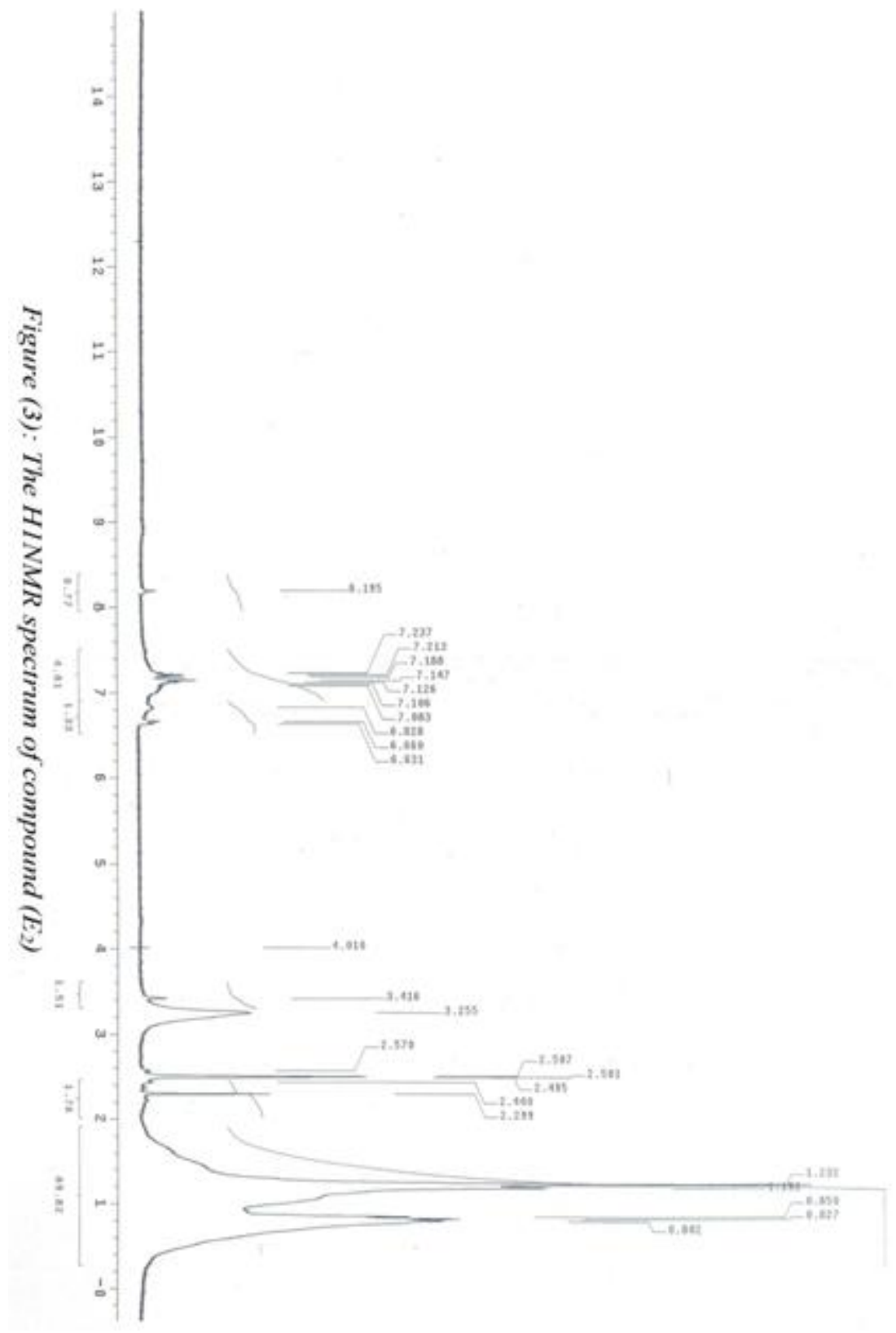




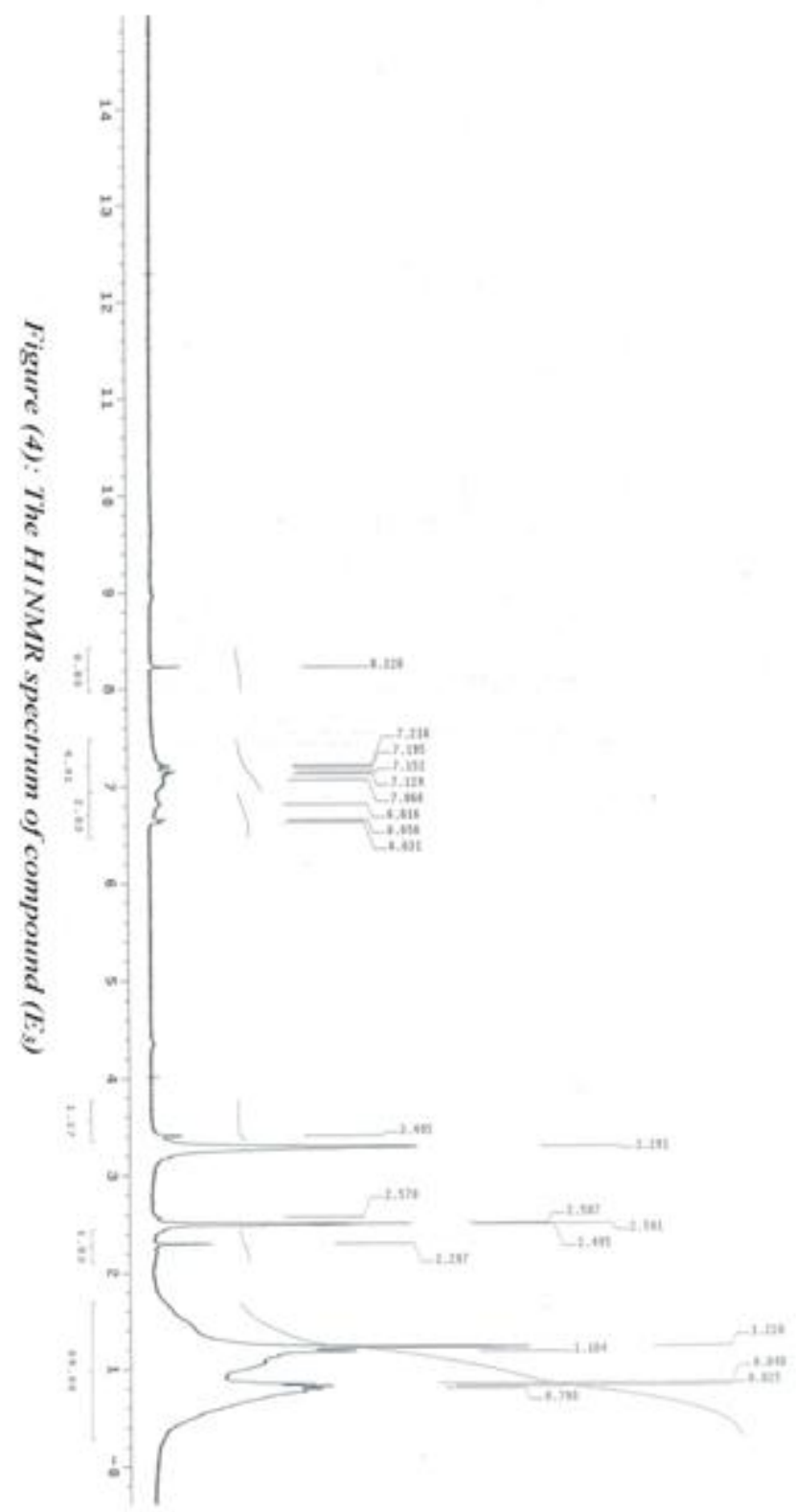

\subsection{Evaluation of the Prepared Compounds as lubricating oil additives:}

\subsubsection{As Antioxidants.}

All the prepared compounds were added to a sample of lube oil "SAE-30" free from any additives, in $2 \%$ concentration, and the blends obtained were subjected to serve oxidation condition as described previously and the oxidation stability expressed as increase in viscosity ratio (V/Vo) and total acid number (T.A.N.), and optical density (Log I/Io) that compared with lube oil sample free from additives, the results are shown in Figs. $(5-7)$. 


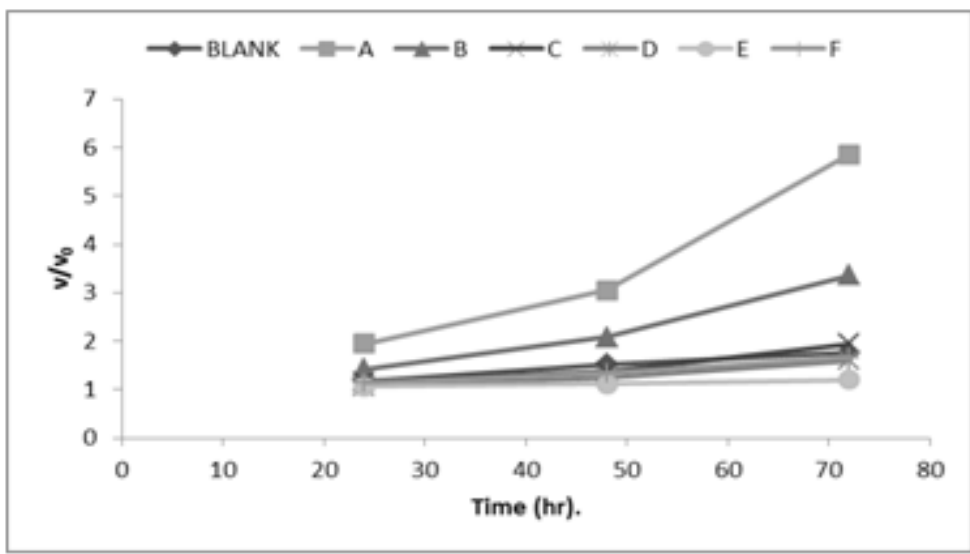

Figure (5): Variation of V/Vo with oxidation time of lube oil without and with additives (A, B, C, D, E and F).

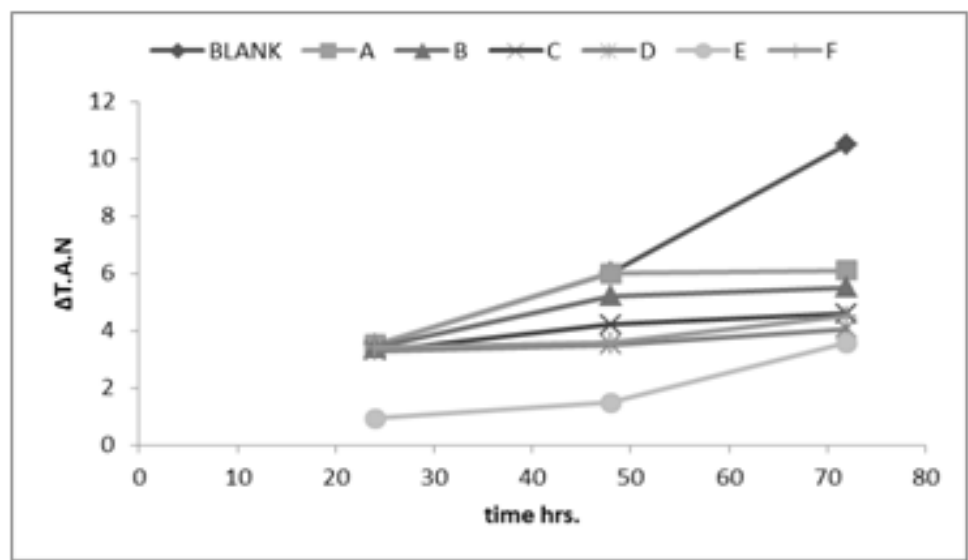

Figure (6): Variation of $\Delta$ T.A.N. with oxidation time of lube oil without and with additives (A, B, C, D, E and F).

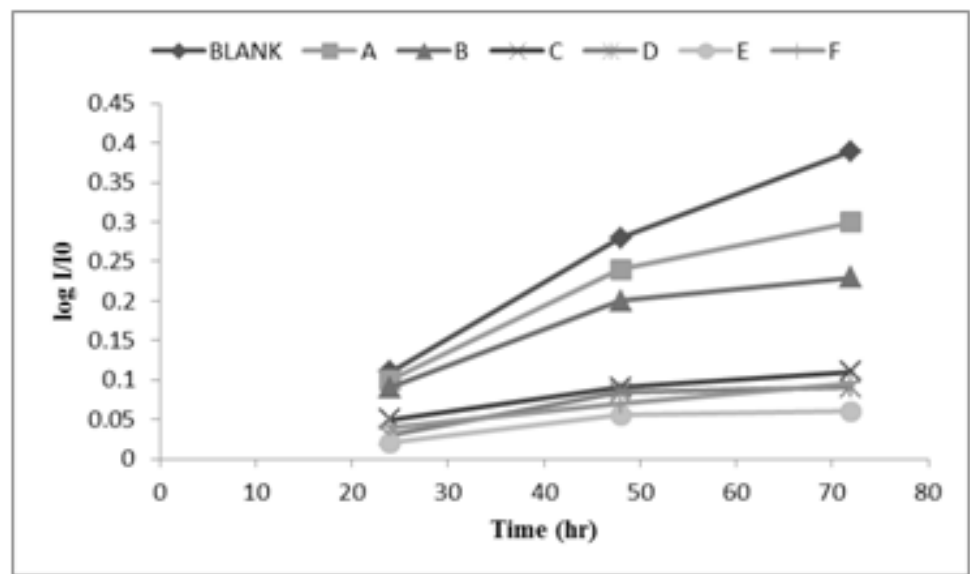

Figure (7): Variation of Log I/Io with oxidation time of lube oil without and with additives (A, B, C, D, E and F).

Inspection of the results obtained in Figs. (5-7) indicate that:

The oxidation stability of the additives prepared increases with the increase of their sulfur content up to $1.5 \%$ sulfur \& then decreases again. It is clear from Figs. (5-7) that additive (E) which has been prepared using a molar ratio of sulfur to phenol 1.5: 1 is the best one in this respect. Introducing sulfur in the structure of the phenates leads to increasing in their antioxidant properties and this may be attributed to the fact that they inhibit oxidation by reduction of peroxides beside the inhibition through chain breaking resulting from the reaction. The reaction of sulphurized phenates prevent oxidation through two mechanisms: chain breaking and peroxide decomposition, while non sulphurized metal phenate act only as chain breaking agents. 


\section{Effect of using different ratios of calcium hydroxide.}

The previous results show that compound $(\mathrm{E})$ gives better results so we prepared the basic and over basic $\left(\mathrm{E}_{2}\right.$, $\mathrm{E}_{3}$ ) of the same compound. The T.B.N. of the prepared compounds are shown in Table (4)

Table (4): T.B.N. of the prepared compounds

\begin{tabular}{|l|l|}
\hline Compound & T.B.N. \\
\hline E & 55 \\
\hline E2 & 102.2 \\
\hline E3 & 277.9 \\
\hline
\end{tabular}

The results of additives with different calcium hydroxide ratios are given in Figs. (8-10), it was found that the additives prepared with higher calcium hydroxide ratio (overbasic $\left(\mathrm{E}_{3}\right)$ ) are more efficient as antioxidant than other prepared additives this may be due to the presence of alkaline calcium carbonate which can neutralize acidic compounds formed during oxidation of lube oil. Increasing the proportions of metal in the phenate, increase their antioxidant properties and this may be attributed to the fact that excess base in the additives can neutralize the excess of acids formed during oxidation of lubricating oil.

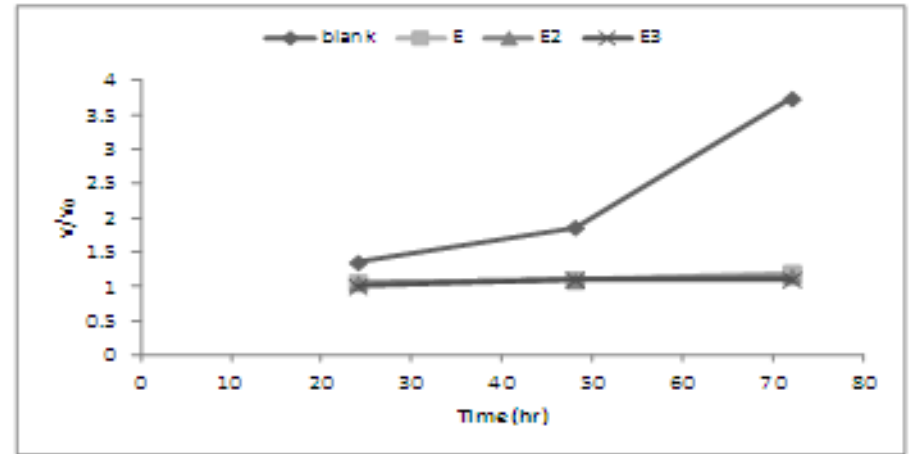

Figure (8): Variation of V/Vo with oxidation time of lube oil with additives of sulfur ratio $=1.5 \%$ with different basicity $\left(\mathrm{E}=\right.$ neutral, $\mathrm{E}_{2}=$ basic and $\mathrm{E}_{3}=$ overbasic).

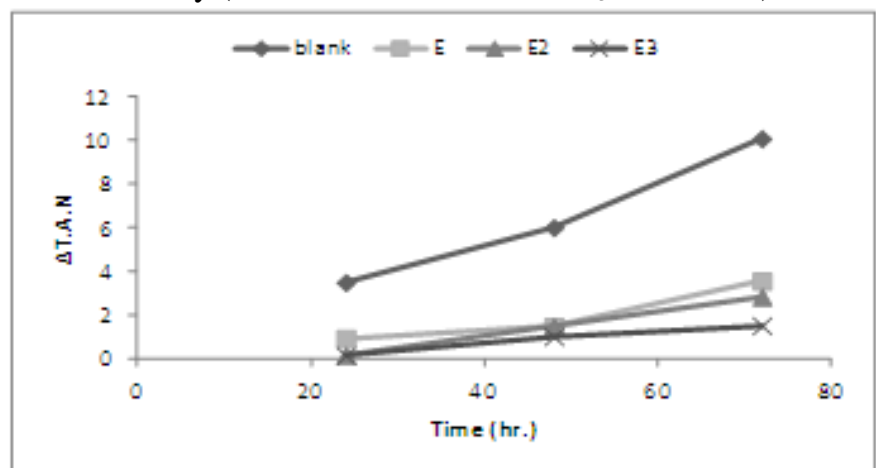

Figure (9): Variation of T.A.N. with oxidation time of lube oil with additives of sulfur ratio $=1.5 \%$ with different basicity $(\mathrm{E}=$ neutral, $\mathrm{E} 2=$ basic and $\mathrm{E} 3=$ overbasic

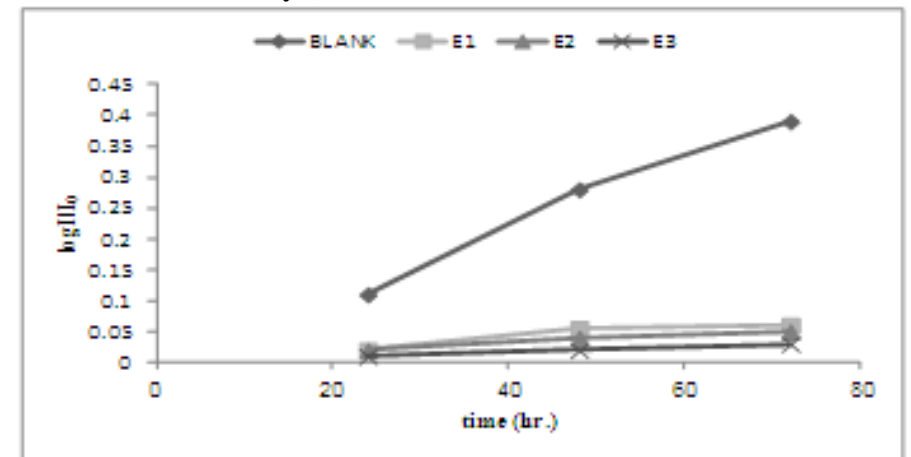

Figure (10): Variation of Log I/Io with oxidation time of lube oil with additives of sulfur ratio $=1.5 \%$ with different basicity $\left(\mathrm{E}=\right.$ neutral, $\mathrm{E}_{2}=$ basic and $\mathrm{E}_{3}=$ overbasic $)$. 


\subsubsection{As Detergents/Dispersants.}

All the prepared compounds have been added to the oil samples in concentration of $2 \% \mathrm{wt}$, using spot test method results given in Table (8) show clearly that the prepared compounds have very good and excellent dispersion power (61-97\%) for sludge and solid particles formed during lube oil oxidation compared with lube oil free from additives [14]. It is clear that the oxidation of these compounds disperses solid particles in the oil thus prevent their agglomeration and participation on metallic parts of engines.

Table (5): Percentage of dispersion (spot test method) of the lube oil sample and it is blends with additives after different oxidation time (hrs.).

\begin{tabular}{|c|c|c|c|}
\hline \multirow{2}{*}{ sample } & \multicolumn{2}{|c|}{ Percentage of dispersion, time hrs. } \\
\cline { 2 - 4 } & $24 \mathrm{hrs.}$ & $48 \mathrm{hrs.}$ & $72 \mathrm{hrs}$. \\
\hline blank & 35 & 33 & 32 \\
\hline A & 80 & 83 & 85.06 \\
\hline B & 70 & 91.35 & 90.24 \\
\hline $\mathbf{C}$ & 90.38 & 94 & 93.88 \\
\hline $\mathbf{D}$ & 80.77 & 84.31 & 95.56 \\
\hline $\mathbf{E}$ & 90.2 & 95 & 97.06 \\
\hline $\mathbf{F}$ & 88.46 & 88.89 & 90.74 \\
\hline $\mathbf{E}_{\mathbf{2}}$ & 91.49 & 89.36 & 86.67 \\
\hline $\mathbf{E}_{\mathbf{3}}$ & 89.58 & 89.8 & 88.24 \\
\hline
\end{tabular}

The results of numerous experiments attested that these two methods were suitable to estimate the potential detergent/dispersant efficiency (PDDE \%) in oil solutions:

$$
\mathrm{PDDE}=\frac{\mathrm{DI}+\mathrm{WE}}{225} \times 100
$$

Where DI is the detergent index (\%), WE is the washing efficiency $(\mathrm{mm}), 225$ is the maximum value of DI + WE $($ DI $\max =100$, WE $\max =125)$

The results are shown in Table (6).

Table (6) Percentage of potential detergent/dispersant efficiency (PDDE) of the prepared compounds

\begin{tabular}{|c|c|}
\hline Sample & PDDE \% \\
\hline A & 53.78 \\
\hline B & 80.64 \\
\hline C & 81.8 \\
\hline D & 85.56 \\
\hline E & 87.94 \\
\hline F & 77.8 \\
\hline $\mathbf{E}_{2}$ & 88 \\
\hline $\mathbf{E}_{3}$ & 91.11 \\
\hline
\end{tabular}

It seems that all results give very good and excellent behavior according to potential detergent / dispersant efficiency.

\section{Conclusion}

The conclusions could be derived from the results of this study that:

- Preparation of neutral calcium phenates and their corresponding basic and superbasic additives.

- All the prepared compounds are good soluble in lubricating oil (SAE 30).

- The structures of the prepared compounds were confirmed by (F.T.I.R), (G.P.C) and ( ${ }^{1}$ H-N.M.R).

- The efficiency of the prepared compounds as lube oil additives (antioxidants and detergents/dispersants) was studied. It was found that all the prepared additives have excellent power of dispersion and detergency.

- It was found that the efficiency of the prepared compounds as antioxidants increases with increasing its basicity

\section{References}

[1]. P. Ghost, and M . Das, "Study of the influence of some polymeric additives as viscosity index improvers and pour point depressants"; J.Petrol.Sci.Tech., 119(9), 2014,79-84.

[2]. X. Fernández-Pérez. , A. Igartua, R. Nevshupa and P. Zabala, Tribology - Fundamentals and Advancements: "Innovative "Green" Tribological Solutions for Clean Small Engines"; (Tribol.Int 2011), 727-736.

[3]. [3] S. George., S.Balla and M.Gautam, "effect of diesel soot contaminated oil on engine Wear"; Wear, 262(9), 2007, 1113-1122. 
[4]. J. Booth, "surface engineering and tribology for future engines and drive lines"; Advances in Electrostatic Health Monitoring of Ttribocontacts conference proceedings of 50th IMechE tribology group anniversary conference, 2006

[5]. J. Braun., T. Mang and W. Dresel, "Lubricants and lubrication Additives"( $2^{\text {nd }}$ Ed), (Wiley-VCH GmbH, Weinheim 2007) 88-100.

[6]. V. Duhalt. R, "Environmental impact of used motor oil"; Sci .Total. Environ, 79(1), 1989,1-23.

[7]. S.P. O'Connor, J.Crawford, and C.Cane, "Overbased lubricant detergents - a comparative study"; Lubr .Sci., 6(4), 1994, $297-325$.

[8]. B. Besergil, A. Akin, and S. Celik, "Determination of Synthesis Conditions of Medium, High, and Overbased Alkali Calcium Sulfonate"; Ind .Eng .Chem .Res, 46(7), 2007, 1867-1873.

[9]. J. Galsworthy, S. Hammond, and D. Hone, "Oil-Soluble Colloidal Additives"; Colloid .Interface Sci, 5(5-6), 2000, $274-279$.

[10]. D. C. Hone., B. Robinson, and D. Steytler, "Mechanism of Acid Neutralization by Overbased Colloidal Additives in Hydrocarbon Media"; Langmuir, 16(2), 2000, 340-346.

[11]. G. Lamb, C. Loane, and J. Gaynor, "Indiana Stirring Oxidation Test for Lubricating Oils"; Ind. Eng. Chem , 13(5), 1941, $317-321$.

[12]. V.A. Gates, R. F. Bergstrom, and L.A.Wendt, "Further Discussion on "Oil Spot" Evaluation of used Engine Lubricants"; Society of Automative Engineers (SAE)",paper 550126,1955.

[13]. J. Hancsok., M. Bubálik, and A. Beck, "Development of multifunctional additives based on vegetal oils for high quality diesel and biodiesel"; chem. Eng. Res, 86(7), 2008, 793-799.

[14]. N. S. Ahmed., A. M. Nassar and R. S. Kamal, "Influence of Some Compounds as Antioxidants and Detergents/Dispersants for Lube Oil"; J. Disper. Sci. Technol, 32(7), 2011, 1067-1074. 